\title{
Step by Step in Anatomic and Diagnostics Veterinary Pathology: How Death Teaches Us about Life
}

\author{
Peter Makovicky $^{1 *}$, Jacqueline M. Arnone ${ }^{2}$, Pavol Makovicky $^{3}$ \\ ${ }^{1}$ Laboratory of Veterinary Histopathology in Komarno,Slovak republic. \\ ${ }^{2}$ School of Nursing, Kean University, Union New Jersey, U.S.
}

${ }^{3}$ Selye Janos University, Pedagogical Faculty, Department of Biology, Komarno, Slovak Republic.

\begin{abstract}
Veterinary pathology is an interdisciplinary part of veterinary medicine. In much of society, it is still a prevailing opinion that this work is dedicated only to the dead animals. However this is not true. First of all it is a diagnostic discipline. It has broad relevance for clinical practice, almost for all clinical fields, including preventive results and results are applied also in wider medical research. The authors are trying to describe the veterinary pathology way, as a way from death to the work with living and to showing how attractive veterinary pathology can be. The full text is written in an unusual form using some of our own experiences in the veterinary pathology work. The main objective of this work is to comment and then briefly list the scope of work of a veterinary pathologist. The text is written in an atypical form with the express purpose of attracting readers who have never been in direct contact with a veterinary pathologist before and give them a better understanding of the profession. In this sense, there are informally selected past personal vignettes and some aspects of veterinary pathologist work.
\end{abstract}

Keywords: Biopsy, Cytology, Histopathology, Immunohistochemistry, Molecular biology, Necropsy, Veterinary pathology.

\section{The Theory ANd How IT Was ACtually StaRted?}

I remember quite well, when I was a young student attending the university. Veterinary pathology was, at that time, predominantly perceived as one of the fields, which primarily focused on working with dead animals. Practical exercises were conducted at a department's necropsy room, which was localized in the corner of the areal. Early morning exercises forced me to get up very early, and in the winter it was still dark outside as I waited in front of a semi-glass building. There were rather smaller autopsy rooms, which at first gave me an inhospitable impression and the static approach of pedagogues enhanced the cold-hearted atmosphere. To hide was not possible, because the room was filled by four shorter and consecutively ragged, blue painted wood benches, where in one row near each other they were fitted with four, maximally five students. In front of the first row, was a simple marble autopsy table that was approximately two meters in size and behind that table about three meters away, was the second autopsy table. Behind the second autopsy table was a large glass wall, covered with grey curtains, overlooking the outer courtyard. The walls were half covered by white tiles and all sides were stacked by shelves. On them were many differently sized glass jars with organs, and bottles with whole animals in fixation, with lifeless dull stares. On the top of that shelf were simple rods that were attached to the skeletal preparations of various animals, especially livestock. The flimsy skeletons looked as if they would fall down at any time or totally fall apart. A large part of them were damaged, due to systematic manipulation, which had impaired the joints between the rib bones, broken vertebrae and broken limbs with missing bones. Some were missing the entire larger bones and sometimes even the skull itself was missing. On the walls were various black and white posters with different drawings and mass of arrows, briefly referring to the professional Latin terminology for each body part.

\section{Practical Exercises and how it All Continued?}

The work was conducted with an emphasis on strict discipline. Student filter was, at that time, a rather imaginary space and our personal things were left in the histologic room. After entering the autopsy 
room, it was compulsory to wear a white buttoned lab coat at all times. Long hair had to be tied back, and no one could have rings or other decorations on their hands. Most importantly, it was imperative to report any injuries sustained while we were working. Working with biological material was allowed, but only with safety gloves. The course itself was not very entertaining, and rather resembled a dictionary of foreign words which was necessary for us to already know during a particular exercise. There were interpreted terms and relationships between meanings, and step by step procedures during different situations. All of these things were visually supported by specific cases. Most cases involved animals from a school farm. I remember one time I was ordered to dissect a dead sheep from an autopsy assistant. During the opening of the thoracic cavity, I encountered a problem, which I tried to compensate by physical force under the strict time pressure. I took a classical axe and a medium sized hammer. I had cut through a centre of the ribs at a right angle, in a bit of a semi-chaotic way. Let me explain to further understand my dilemma. Due to a non-ideal position of the hammer, the hits were made towards the ribs, causing a bounce back. It looked like the hammer was constantly rebounding, or, in the better case, it happened a crack and subsequently crush the ribs. The lungs had a bordered purulent granuloma in irregular shape of egg, approximately the size of an adult man's palm. The axe repeatedly penetrated the granuloma, which was a liquid in the centre. The volume of purulence was so great, that it caused it to spew everywhere. So much so, that I even had yellow stains in my hair. I was so immersed in the work, that I did not realize it was in my hair until after the crushing all ribs. I also remember very well the autopsy of an adult boar. We were given the task to skin this animal. I can honestly confirm that it is quite physically demanding work, especially during the preparation of the skin on the legs. This work requires total concentration and the utilization of very sharp instruments. Already after a few minutes of effort, the knife slipped out of my hand and under the continuous pressure, the blade flew away, catching the middle finger of my left hand. I was surprised from the start that the blood did not readily flow from my finger. I admit, that this was not an exemplary action from me, but the resulting miniature wound I ended up with, I sutured back together. I still have this wound as a memory and even during the writing of this text I can see the approximately $1.5 \mathrm{~cm}$ scar on my finger. Once, we got the task to skin some adult silver foxes. But the professor forgot to tell us that he wanted to taxidermy the animals and then sell them. At that time, there was a great demand for those goods. We cut the limbs, including heads, and only a fur remained in a residue of a body. Several times, we even accidentally cut through the residue of the fox fur. We were not absolutely prised for this particular exercise. I also remember another time, when I assisted during the process of euthanasia in order to perform an autopsy on the animal afterwards. Most animals euthanized for autopsy were sick sheep. However, I will add some other stories from the life at the veterinary necropsy. An owner brought in an Arabian mare for euthanasia. The associate professor ordered that the euthanasia should take place in the area of the necropsy room. The mare was markedly, but gently nervous as she entered the necropsy room. During the process of the application of the injection for falling euthanasia, the mare immediately ran against the wall, breaking everything that was there. She galloped towards the benches around the autopsy tables, while the autopsy assistant waited for her from the other side. It might seem rather funny to the reader, but it was not a joke. Another time an owner of a dog diagnosed with progressive chronic disease, asked us to euthanize the dog. The dog was a smaller crossbreed, which was transported directly in necropsy room, as recommended by the associate professor. In our silent presence, our associate professor applied an efficient substance to produce the euthanasia. Shortly after the application, he stated an exitus letalis and put the lifeless body of the dog into a dark plastic bag. To our shocked surprise, the dog started to awaken after few minutes. Under the supervision of an associate professor, we were asked to perform the autopsy of a dead snake with suspicion of cardiovascular failure. After a few cuts, the professor disappeared with an apparent sour facial expression, stating he would return after a few minutes and that we were allowed to work independently. In the end, he did not return, so we finished the autopsy and he only signed the autopsy report. Another time, we were in the area of the necropsy room where there were delivered boxes with several tens of live, wild ducks that were needed for another department. An associate professor ordered, "we have to kill them, blood the mout, remove their feathers and then scald the min warm water and remove the feathers completely. Then all of you wash your hands and dissect them". After the end of work he came back, showing appreciation for our work and then self-confidently said: "This part we will embed into paraffin and the other part we will mix and then embed into paraffin too". I will leave it to each reader's discretion, where it all ended... Another day we received the task from our associate professor to prepare an autopsy for some adult laboratory sewer rats, which we had to anesthetize by a solution of 
chloroform, according to protocol. After explaining and then dividing the tasks, the work started in some small groups of students, without pedagogical supervision. The sewer rats were located in bigger plastic breeding cages. One of the students from one of the groups decided to alter one of the tasks thinking that would make the work easier, or in retrospect, maybe he did not understand the procedural protocol entirely. The student poured the chloroform directly from the bottle into the plastic container. He did not save the solution at all. As a safety measure, he let some time elapse. As a result of his action, all of the rats' bodies were affixed to the bottom and wands of the plastic cages. The bodies were unusable for autopsy, and the cages were permanently damaged. Exceptionally we could help with creation of a permanent mount, which were put "forever" into glass containers. This was long term work, which necessitated going to the necropsy room even in the evening time. Once, during this evening task, the assistant responsible for us doing this task went out of the room. $\mathrm{He}$ poured out several tens of litres of acetone and petrol-alcohol directly on the autopsy table. Everything quickly flowed down through a sink-hole into the main collecting container of the autopsy table. This container was connected to another thinner outflow, which already lead somewhere out into a waste line. The outflow was located approximately in the centre of the head collecting container, so that all of the liquid could not flow out. Moreover, the head collecting container was filled with the remaining flushed material. Imagine the shock of everyone that next day, when a student who was standing near the autopsy table outflow fainted during an exercise. Thatevening we dismantled the autopsy table with an assistant and cleaned the head collecting container. I remember how we cleaned the box freezers. From time to time, but mostly only when the build-up of frost no longer allowed it to be closed, the technicians need to clean the freezer. After defrosting the freezers they left them in that state. All the melted material began to be outgrown by fungus. So after some time, when you opened the box freezer there was a mound of thawed organs floating in spilled blood and everything was overgrown by massive amounts of fungi. If you want to wash it, you need to stoop down yourself. Our technicians begrudgingly went to work, but when stooping down to clean the bottom of the freezer, they began to retch. It presented an interesting sight, as they were young, pretty, long-haired girls, often dressed in a short skirt with long nails.

The second part of the practical exercises in veterinary pathology was taken in the histology room, which was in another part of the same building. It was a relatively reserved, sometimes "sleepy interpretation" by the lector who notified our class that a significantly expensive and relatively slow examination would be given at the end of the course. The lector also announced that we were to be very careful and not to break the microscopic slides. The environment of these clinical exercises was extremely disciplined. The windows were almost always hidden behind dark non-transparent curtains. The work proceeded in absolute silence. Almost all of the lectors were quite strict and vigilant of silence so nothing would disturb us as we worked. Long hair had to be tied, rings and other decorations on our hands was forbidden. We had to repeatedly draw the changes we saw on the slides into our workbooks. For most of us, it was a rather tiring, and non-interesting task and we did it only from duty. For some students, every minute dragged on, yet for others the time went by very fast. During an informal conversation I had one time with a specialist assistant, he honestly admitted to me that he often did not have any idea what kind of tissue was on the training slides. He told me that he always aligned the training slides into a box in a certain order according to numbers, to know what kind of tissue it was. Every exercise began with a reminder of the safety measures and how we needed to be careful with the light microscope and the slides. During one of these demonstrations, a professor had loosened the revolver head of the microscope and the lens fell down directly onto a slide, breaking it apart in ten pieces. In the room, there was an immediate deathlike silence, but our professor said: "So, students, you can see that we all make mistakes". Nevertheless it has been clear to us that veterinary pathology is a very wide and diverse field. It made us amplify the complexity and comprehensiveness of the object studied and increased my natural curiosity and hunger for more information. They commenced with the macroscopic description and then lead to the analysis, and then followed by a deeper analysis at a sub cellular level. The accompanying course supplement was a relatively thick book, and included books with notes that kept us company at night and sometimes longer. Gradually, we found some relationship between the macroscopic and microscopic changes. At times, others assisted us to navigate the material, but there were times that we tried to deduce things myself without simplifying them. In retrospect, we found this not to be too complicated. One of the main routes to a common pilgrimage of veterinary pathology was not to think through, but go step by step and not base findings on unfounded interpretations. 


\section{From Theory to the Practice AND What Connects the Veterinary Pathologist with the Living Population?}

It has already been many years since we left the school gates, and since then there has been a change of view regarding veterinary pathology. It is still a diagnostics field, but far from being only an analysis and synthesis, which provides simple answers to questions of causes of death. The center of veterinary pathology has moved among the living. In this sense, we have to note, that at the start of our practical internal assistance, we had maybe $90 \%$ necropsy practice and the residual $10 \%$ fell below the biopsy practice. Today this ratio, in most countries all over the world, is perhaps in favor of biopsy, but in some places the people doing a final diagnosis from necropsy, are doing so without microscopic investigations. At the time, we did not carry out intra operative biopsy, and the clinical thin needle aspiration cytology was the "great unknown" as well. I remember how the head of the department of veterinary pathology and my supervisor Dr. Lorko one day said to me "Do you really want to achieve something in this field? If yes, do not wait and every day work in the necropsy room. This work will bring you one day among the living and for living”. Today I just can add, that he is right. But there were really big differences between work at this time and today. The work went very slowly, both because of the relatively complicated procedures, along with the manual activity. All solutions, like histological dyes, were needed to do using powders and then mature for some time. Each procedure was done by hand, and included creating boxes for samples, histological cartridges, and the transference of samples from one solution to another. Paraffin had to boil and it was necessary to add various additives to them. Samples were manually bonded to wood pads. Knives were sharpened by hand and those persons who wanted to cut the samples needed to have very good nerves and great interest. Owing to the limited space of this article, we are not going to mention other manual activities, but today is the same work reduced by minimum half and human mistakes are also limited. This is the results of modern semi-automatic, or fully automatic laboratory instruments and welldefined time procedure in combination with a technical approach to working with the samples [1]. This time we personally had considered and realized that the biggest impediment was the very poor professional contact between us and our clinical colleagues. They didn't go to the autopsy room at all and there were only informal meetings. I remember well, how my supervisor Associate Professor Kulisek and I were waiting in the necropsy room to the other clinicians who were invited to witness an animal autopsy. Usually no one attended, or they would send a young graduate assistant, who would stand like a pillar in silence waiting fervently for the autopsy to end. I myself have often wondered what could have been done to improve the professional contact and collegiality between us and other clinical disciplines. Individual professional relationships certainly persisted as a result of ongoing research. Our laboratories possessed instrumentation for the production of permanent microscopic slides. Selected photos were inserted into various scholarly publications, including lectures or textbooks for students. But the work went very slowly, both because of the relatively complicated procedures that are now completely substituted by modern semi-automated and fully automated machines and also on the grounds of absolute indifference of some of the staff on research teams. Our work was predominately geared towards teaching and diagnostics. Research activity was again the domain of specialized institutions, which were by state actors responsible for addressing selected research tasks. Just as an example, here is a paradox. The former research institute has since established a special department that exclusively solved secret research. Everything was top secret, including the commission which approved them, and comprised the findings of these investigations. The results of some of the investigations were disclosed only to the director of the Institute and paradoxically he could not even know about the results of the commission that approved them. The department was located in underground areas where it was not possible to enter, and even if someone somehow got to one of those areas, he would hit the next door of individual cabinets. During an informal conversation at a joint lunch, one former employee told me how most everyone just sat there with nothing to do, only watching what the other was doing. For example, one room regularly echoed through the corridor by the hoarseness of a weary researcher. However, news from abroad began to penetrate the field gradually, so in a few years everything started to change. The veterinary pathology is interdisciplinary field of the veterinary medicine and conception is built on several pillars [2]. Changes related to an interest in living anatomy have also reflected an increased claim of clinical colleagues to veterinary pathologists. This applies to routine clinical practice and in research. Today, 
we certainly do not have enough to write a one word conclusion, but the veterinary pathologist should possess both dynamic analytical and synthetic thinking. In this sense, he should seek out relationships between clinical symptoms, changes in the tissues and the development of the disease itself. Yet he must remain objective and not fabricate the interpretation without material proof. It is still true that veterinary pathology is a strict discipline, a fact which remains open to additional further meaningful and reasonable discussion. I remember my supervisor, Associate Professor Durcansky. He always said "Independently, we must have the same diagnosis, as European, American, Canadian and also Australian pathologists". Additionally, my supervisor Professor Vannucci also always emphasized, "If major differences exist between us (colleagues in the field), we should defend our own conclusions, or finally admit our mistake". It is our hope that this text will also be read by some of our colleagues from other countries, and that they will share our opinion that their results need to be the same. Medical research is ultimately always based on the findings and pathology. This includes covering the results of biopsy and necropsy examinations including detailed descriptions which are based on advanced immuno histochemical and molecular pathological examinations. Many people do not realize that chemotherapeutic agents arose from the findings of those previously listed indicated examinations. Clinical colleagues therefore do not base their decisions according to their own feelings or findings, but also rely on the conclusions made by the pathologist. So pathology becomes an integral part of the diagnostic fields and significantly helps in creating therapeutic procedures. Finally, one piece of evidence to support the advancement of the field is the arrangement of modern research centers, which include veterinary or human hospitals. Today in these settings, the biopsy labs are placed directly in the center of the buildings. This is in sharp contrast to the past when the pathology laboratory was regularly located in isolation somewhere in the corners of the building and literally regarded to as a "funeral home" with the main task of collecting corpses. People who had problems with alcohol or were mentally handicapped, perhaps found a place at the department of pathology. I well very vividly remember on my first workplace immediately after graduating. I arrived with big respect to the department of veterinary pathology asking for a job. The head of the department said first "I have on you only one important request: Please do not drink a lot of alcohol, so you may be drunk, but please do not wallow yourself here". I was a disappointed in that welcome, but I was accepted. In our opinion, this field this time remains interesting and attractive to clever and intelligent graduates, young colleagues and worldwide professionals who work in this field. Contemporary veterinary pathologists today, "really do not step the animal corpses". Our work is mainly for the living, for preventive practice, and research. A similar trend includes necropsy, even when conclusions of all veterinary pathology institutions across the state gather and then organize the ladder of causes of death of individual animals as well as the prevalence of diseases in specific animal breeds. By creating a hierarchy, the general public might better understand what diseases are the main causes of death of domestic animals. This would serve as a benevolent and useful tool for our clinical colleagues. For example during a first preventive health show, an animal's owner could ask questions regarding breeding the different breeds of animals to avoid future disease. Furthermore, knowledge could be shared regarding what prognostic significance had diagnosed the disease, how the disease is likely to develop further, or how to answer the question whether it is possible or not to cure the animal. Correct necropsydiagnosis is also important for the animal husbandry breeders. For example one important case from our necropsy practice: our post-mortem results show that a frequent killer of animal husbandry is poor nutrition. Or another view, correct necropsy diagnosis can protect people before a dangerous animal husbandry health infection occurs, which can infect food like milk, or the histopathological results can check the quality of the meat $[3,4,5,6]$. Diagnosis also protects other animals before they come into contact with infected animals. It is clear that necropsy work is very important for the living, animal husbandry, wild animal populations, for farmers and also for people who eat meat and milk products. This work isnot static or simple mechanical work. I will give one more example. I remember back to my short praxis in Pathology and parasitology department. As I was starting a dead pigletautopsy, the head of the Institution and my supervisor Dr. Mydlo said me, "Some people think that we are just going to cut meat, but in fact, only now everything begins. At the end we need to give realistic and useful results, because the requester will not pay for our work". Finally you can see that some farmers are already well aware that veterinary pathologists are not butchers. The veterinary pathologist's work is, in this meaning, very important in collaborating with other cohorts such as biology, parasitology and chemistry. 
Today for all interested, itis obvious that the future of veterinary pathology is hand in hand and associated with genetic and molecular biological research. It is known that the function and importance of individual genes contained in the animal cell are in context with themselves evolution, development and program parameters of life. While today we are all fully aware that many illnesses arise on the basis of genetic predisposition and, therefore, a way to repair genes is one of the preferred routes of therapy in modern medicine. We believe that we need to know more about the functions of individual genes and their specific roles. Then we can provide insights into the pathophysiology of disease and better understand the mechanism of them. The actual pathology here undoubtedly still holds an important place in this field[7]. First in the diagnostic role, if the sequence supports or refutes the findings, including detailed descriptions documenting these changes is warranted. Furthermore, genetic sequence is also important in application and therapeutic levels. For example, in human medicine, there is already a whole spectrum of procedures to date that are able to detect a predisposition to various diseases, including oncological diseases. Here we would like strongly recommend to every young people interesting in this field, to participate in specialized veterinary pathology training.

\section{Conclusion}

We started with an autopsy table, and even today it surely remains, but the scope of work of a veterinary pathologist is much broader than it appears at first glance. Veterinary pathology is still here for the living population. During our student experiences it has been demonstrated that many things remain unclear. Some are perhaps better studied, but others are unclear, both in the context of the clinic and the development of changes at the microscopic level. Thus, there remain a number of unanswered issues. We believe that one way to become closer to these answers is based on implementing a thorough study of the existing information about a specific disease, setting targets and supply of material with good instrumentation, laboratory facilities and personnel. Active interdisciplinary cooperation with colleagues in biology, chemistry and possibly other related fields would then also be applied with results affecting clinical practice. Outcomes are also one of our goals, and other relevant evidence that veterinary pathology is here for the living. Our main objective is still to yield a quality diagnosis, by which those diagnoses can be reliably shared with our clinical colleagues. This would include the addition of information on special examinations which are useful in prognosis of disease, therapy, cause of illness, and more importantly disease prevention. We also want to create quality outcomes for all researchers who work with us. The results of our work stays behind the variable success or other practical export, or are a classical part of lectures of other colleagues. These are the objectives for all veterinary pathologists worldwide and we will be stronger and more unified as a discipline only if we cooperate and collaborate together all over the world. Then we can solve important problems that in the end will ultimately contribute to a better life for animals and humans globally. We humbly believe that our message will be an example for all of our colleagues both in the present and beyond in the future.

\section{DEDiCATION}

This article received no specific grant from any funding agency in the public, commercial and any other private profit sectors.

\section{REFERENCES}

[1] Kanchev I., Makovicky P., CCP histopathology in action, Phenogenomics Newsletter. 1(1), 1011 (2015).

[2] Makovicky P., What does modern veterinary pathology have to offer? J. Anim. Vet. Sci. 1(1), 43-47 (2015).

[3] Makovicky P., Nagy M., Makovicky P., Szinetar Cs., Milk quality comparison of the sheep breeds (Improved Valachian, Tsigai, Lacune) and their crosses, Magyar Allat. Lapja. 135(2), 8590 (2013).

[4] Makovicky P., Milerski M., Margetin M., Makovicky P., Nagy M., Genetic parameters for the size of uder cisterns in ewes diagnosed by ultrasonography among breeds: Improved Valachian, Tsigai, Lacaune and their crosses, Arch. Zootec. 64(248), 403-408 (2015). 
[5] Makovicky P., Makovicky P., Levkut M., Chrenkova M., Kulisek V., Jilek F., Histopathological and genetic aspects of stress syndrome of the skeleton musculature of pigs with resulting PSE meat, Folia Vet. 51(3-4), 185-188 (2007).

[6] Vadlejch J., Makovicky P., Cadkova Z., Langerova I. Efficacy and persistent activity of moxidectin against natural Muellerius capillaris infection in goats and pathological consequences of muelleriosis, Vet. Parasitol. 218, 98-101 (2016).

[7] Makovicky P., Svecova I.,Veterinary pathology: the past, present and the future, Phenogenomic Newsletter. 2(2), 22-23 (2016). 